\title{
Trellis-Based Joint Huffman and Convolutional Soft-Decision Priority-First Decoding
}

\author{
Yuh-Ming Huang ${ }^{1}$ and Yunghsiang S. Han ${ }^{2}$ \\ 1. Department of Computer Science and Information Engineering, National Chi Nan University, Puli, Taiwan \\ 2. Graduate Institute of Communication Engineering, National Taipei University, Taipei County, Taiwan \\ (e-mail:ymhuang@csie.ncnu.edu.tw yshan@mail.ntpu.edu.tw)
}

Let $\boldsymbol{A}=\left\{a_{1}, a_{2}, \ldots, a_{26}\right\}$ be a set of 26 English alphabets with probabilities $\boldsymbol{P}=\left\{p_{1}, p_{2}, \ldots, p_{26}\right\}$ and $\boldsymbol{C}$ be the corresponding Huffman code that has 26 codewords $\left\{\boldsymbol{c}_{1}, \boldsymbol{c}_{2}, \ldots, \boldsymbol{c}_{26}\right\}$ with average codeword length 4.15573. The respective lengths of the codewords are given by $\left\{\ell_{1}, \ell_{2}, \ldots, \ell_{26}\right\}$. According to $\boldsymbol{P}$, a concatenation of 1000 symbols randomly generated from $\boldsymbol{A}$ is Huffman encoded into a bitstream $\boldsymbol{x}$ of length $M$ and $\boldsymbol{x}$ is further encoded into a bitstream $\boldsymbol{y}$ of length $N$ by a binary $(2,1,6)$ convolutional code with octal generator sequence 554 and 744. Then, $\boldsymbol{y}$ is BPSK modulated and sent over an AWGN channel. Let $\boldsymbol{r}=\left(r_{1}, r_{2}, \ldots, r_{N}\right)$ denote the received bitstream. This work assumes that no bits are deleted by the channel, and that the side information $N$ is known at the receiver.

The transmitted bitstream $\boldsymbol{y}$ or its corresponding bitstream $\boldsymbol{x}$ can be represented as a path in a symbollevel trellis. For each node $(l, s)$ in the trellis, $l$ denotes a position (level) in the bitstream $\boldsymbol{x}$ following a valid codeword [1] and $s$ denotes the current register state of the binary convolutional encoder. The codeword, that presents a symbol, or its convolutionally encoded bitstream labels each branch in the trellis. Let $S_{N}$ denote the set of all valid bitstreams of length $N$ formed by the outputs of the convolutional encoder for sequences of codewords in $\boldsymbol{C}$. For maximum a posteriori (MAP) decoding, a bitstream $\hat{\boldsymbol{y}} \in S_{N}$ needs to be selected such that

$$
\operatorname{Pr}(\boldsymbol{r} \mid \hat{\boldsymbol{y}}) \operatorname{Pr}(\hat{\boldsymbol{y}}) \geq \operatorname{Pr}(\boldsymbol{r} \mid \boldsymbol{y}) \operatorname{Pr}(\boldsymbol{y}) \Leftrightarrow \sum_{j=1}^{N}\left(h_{j} \oplus \hat{y}_{j}\right)\left|\phi_{j}\right|-\ln \operatorname{Pr}(\hat{\boldsymbol{y}}) \leq \sum_{j=1}^{N}\left(h_{j} \oplus y_{j}\right)\left|\phi_{j}\right|-\ln \operatorname{Pr}(\boldsymbol{y})
$$

for all $\boldsymbol{y} \in S_{N}$, where $h_{j} \triangleq\left\{\begin{array}{ll}1, & \text { if } \phi_{j}<0 \\ 0, & \text { otherwise }\end{array}\right.$ and $\phi_{j} \triangleq \ln \frac{\operatorname{Pr}\left(r_{j} \mid 0\right)}{\operatorname{Pr}\left(r_{j} \mid 1\right)}$. Under the memoryless source and channel assumptions, for branch labeled by $\boldsymbol{y}_{l}^{i}=\left(y_{l}^{\left(i_{1}\right)}, y_{l}^{\left(i_{2}\right)}, \ldots, y_{l}^{\left(i_{2 \ell_{i}}\right)}\right)$ between level $l-\ell_{i}$ and level $l$ in a trellis, the new branch metric associated with it is defined as

$$
M\left(\boldsymbol{y}_{l}^{i}\right) \triangleq \sum_{j=1}^{2 \ell_{i}}\left(h_{2 l-2 \ell_{i}+j} \oplus y_{l}^{\left(i_{j}\right)}\right)\left|\phi_{2 l-2 \ell_{i}+j}\right|-\ln \operatorname{Pr}\left(\boldsymbol{y}_{l}^{i}\right) .
$$

Equipped with equation (2), based on a code trellis rather than on a code tree, we can modified the algorithm given in [2] to obtain a joint Huffman and convolutional priority-first decoding algorithm (PFDA). In order to lower the decoding complexity, one heuristic policy was adopted on PFDA to ensure that the level value of the ending node in the next searched path was within 10 to that of the ending node in the farthest visited path. Simulation results indicate that the PFDA provides nearly the same performance as that in [1] while exhibiting a significantly lower complexity (average (ave) and maximum (max) number of branch metric computations) as shown in the following table ( $S N R_{b}$ denotes the signal-to-noise ratio

\begin{tabular}{|c|c|c|c|c|c|c|c|c|c|c|c|c|c|c|}
\hline$S N R_{b}$ & \multicolumn{2}{|c|}{$2 \mathrm{~dB}$} & \multicolumn{2}{|c|}{$3 \mathrm{~dB}$} & \multicolumn{2}{|c|}{$4 \mathrm{~dB}$} & \multicolumn{2}{|c|}{$5 \mathrm{~dB}$} & \multicolumn{2}{|c|}{$6 \mathrm{~dB}$} & \multicolumn{2}{|c|}{$7 \mathrm{~dB}$} & \multicolumn{2}{|c|}{$8 \mathrm{~dB}$} \\
\hline " method & ave & $\max$ & ave & $\max$ & ave & $\max$ & average & $\max$ & ave & $\max$ & ave & $\max$ & ave & $\max$ \\
\hline [2] & 5599109 & 5668494 & 5599109 & 5668494 & 5599109 & 5668494 & 5599109 & 5668494 & 5599109 & 5668494 & 5599109 & 5668494 & 5599109 & 5668494 \\
\hline proposed & 1123514 & 1327974 & 356028 & 434876 & 113470 & 133648 & 50308 & 58203 & 32652 & 34610 & 27662 & 28420 & 26343 & 26735 \\
\hline
\end{tabular}
per information bit).

\section{REFERENCES}

[1] K. Lakovic, J.Villasenor, and R. Wesel, "Robust joint huffman and convolutional decoding," in Proc. of the IEEE VTC, Sep. 1999, pp. 2551-2555.

[2] Y. S. Han, P.-N. Chen, and H.-B. Wu, "A maximum-likelihood soft-decision sequential decoding algorithm for binary convolutional codes," IEEE Trans. Commun., vol. 50, no. 2, pp. 173-178, Feb. 2002. 